\title{
Terra and Aqua MODIS inter- comparison using LEO-GEO double difference method
}

Tiejun Chang, Xiaoxiong Xiong, Amit Angal

Tiejun Chang, Xiaoxiong Xiong, Amit Angal, "Terra and Aqua MODIS intercomparison using LEO-GEO double difference method ," Proc. SPIE 10785, Sensors, Systems, and Next-Generation Satellites XXII, 107851G (25 September 2018); doi: 10.1117/12.2325375

SPIE. Event: SPIE Remote Sensing, 2018, Berlin, Germany 


\title{
Terra and Aqua MODIS inter-comparison using LEO-GEO double difference method
}

\author{
Tiejun Chang ${ }^{1}$, Xiaoxiong (Jack) Xiong ${ }^{2}$, Amit Angal $^{1}$ \\ ${ }^{1}$ Science Systems and Applications, Inc, Lanham, MD 20706. \\ ${ }^{2}$ Sciences and Exploration Directorate, NASA/GSFC, Greenbelt, MD 20771.
}

\begin{abstract}
The Moderate Resolution Imaging Spectroradiometer (MODIS) onboard the Terra and Aqua satellites have successfully operated since their launch in 1999 and in 2002, providing more than 18 and 16 years of continuous global observations, respectively. The inter-comparison between the two MODIS instruments can be very supportive for the instrument calibration and uncertainty assessment. Aqua and Terra MODIS have almost identical relative spectral response, spatial resolution, and dynamic range for each band. Therefore, a site dependent correction for a sensor spectral band pair is not necessary for their comparison. However, Terra is in the morning orbit with an equator crossing time of 10:30 am, and Aqua is in the afternoon orbit with equator crossing time of $1: 30 \mathrm{pm}$. Consequently, there is a dearth of simultaneous nadir overpasses (SNOs) between the two satellites. Major challenges in cross-sensor comparison of instruments on different satellites include differences in observation time, solar angle, and view angle over selected pseudo-invariant sites. In this work, the inter-comparisons of thermal emissive bands are performed over a pseudo-invariant target, using the observations from a sensor onboard a geostationary satellite as a bridge. Himawari8 was launched on October 7, 2014. The Advanced Himawari Imager (AHI) onboard Himawari8 can be used as a reference to bridge the comparison between Terra and Aqua MODIS. AHI has 16 channels; with spatial resolutions from 0.5 $\mathrm{km}$ to $2 \mathrm{~km}$ at nadir and produces a full disk observations every 10 minutes. The band spectral coverage matchup, comparable spatial resolution and near-simultaneous observation between MODIS and AHI provide feasibility to implement a double difference method. This comparison method minimizes the impact of the difference in observation time and solar angle. The comparison results will be used as an assessment for MODIS instrument calibration and will be helpful for future enhancement of the L1B product.
\end{abstract}

Index Terms - Inter-comparison, MODIS, TEB, Radiometric calibration, Himawari8, AHI

\section{INTRODUCTION}

The Moderate Resolution Imaging Spectroradiometer (MODIS) instruments onboard the Terra and Aqua satellite have successfully operated since their launch in 1999 and in 2002, providing more than 18 and 16 years of continuous global observations, respectively $[1,2]$. These global observations have resulted in generation of an unprecedented amount of science data products [3-11]. The inter-comparison between the two MODIS instruments can be very supportive for the instrument calibration and uncertainty assessment. Aqua and Terra MODIS have near-identical relative spectral response, spatial resolution, and dynamic range for each band. As a result, the uncertainties associated with site-specific corrections for a mismatch in these parameters are significantly reduced. Observations from simultaneous nadir overpasses (SNO) between two polar orbiting satellites and use of ground reference have been extensively used [12-15]. However, Terra is in the morning orbit with an equator crossing time of 10:30 am, and Aqua is in the afternoon orbit with equator crossing time of 1:30 pm. Due to the lack of SNO between the two MODIS instruments, the inter-comparison of the thermal emissive bands (TEB) is particularly challenging. Major challenges in cross-sensor comparison of instruments on different satellites include differences in observation time, solar angle, and view angle over selected pseudo-invariant sites. To achieve a high precision inter-comparison between instruments with different observation time is particularly challenging for the TEB.

In this work, the inter-comparisons of MODIS TEB are performed over ground targets, using the observations from a sensor geostationary (GEO) satellite as a bridge. Double difference has been previously used for LEO-LEO comparison [12]. The reference used in this paper is Advanced Himawari Imager (AHI) onboard the Himawari8 geostationary satellite. A double difference method is applied to assess the brightness temperature (BT)

Sensors, Systems, and Next-Generation Satellites XXII, edited by Steven P. Neeck, Philippe Martimort, Toshiyoshi Kimura, Proc. of SPIE Vol. 10785, 107851G · @ 2018 SPIE · CCC code: 0277-786X/18/\$18 · doi: 10.1117/12.2325375 
measurements between the two MODIS instruments using AHI. Himawari8 was launched on October 7, 2014. AHI has 16 channels with spatial resolutions from $0.5 \mathrm{~km}$ to $2 \mathrm{~km}$ at nadir and produces full disk observations every 10 minutes.

In comparison with the previous imagers onboard geostationary satellites, AHI provides more spectral channels, higher spatial resolution, and enhanced radiometric calibration accuracy. The band spectral coverage matchup, comparable spatial resolution and near-simultaneous observation between MODIS and AHI provide feasibility to implement a double difference method. This comparison method minimizes the impact of the difference in observation time for scenes with similar viewing conditions. MODIS repeats its orbit every 16 days. A limited sample number can increase the comparison uncertainty. In this work, MODIS measurements at all view angles are used for comparison and a correction to minimize the effect of view angles is derived.

\subsection{MODIS}

\section{BACKGROUND}

MODIS has 16 TEB, covering the mid-wave infrared (MWIR: bands 20-25) and long-wave infrared (LWIR: bands 27-36) spectral regions. All TEB are located on two cold focal plane assemblies (CFPAs): a short-wave and midwave infrared (SMIR) FPA and a long-wave infrared (LWIR) FPA. The CFPAs are nominally controlled on-orbit at $83 \mathrm{~K}$ using a passive radiative cooler. The on-board blackbody (BB) serves as the primary calibration source and the space view (SV) provides a reference for the instrument background and offsets. The BB temperature is measured using a set of 12 thermistors. For nominal operations, the BB temperature is set at 290K for Terra MODIS and 285K for Aqua MODIS. MODIS TEB calibration uses a quadratic calibration algorithm on a scan-by-scan basis for each TEB detector [16]. During the BB warm-up cool-down (WUCD cycles), the BB temperature varies from instrument ambient temperature $(\sim 270 \mathrm{~K})$ to $315 \mathrm{~K}$. The BB WUCD is used to characterize the on-orbit change in the instrument's nonlinear response coefficients. The linear coefficient of the response function for each TEB is calibrated scan-by-scan with the nonlinear coefficient and offset from an offline lookup table (LUT) derived from the quarterly WUCD events. In this paper, collection 6.1 L1B data is used for the comparison. Terra photovoltaic (PV) LWIR cross-talk has been corrected for bands 27-30 for collection 6.1[17]. To reduce seasonal fluctuation effects, the entire year of 2017 data are analyzed for the comparison.

\subsection{AHI}

The Himawari8 spacecraft was launched on October 7, 2014 at an altitude of $\sim 35,800 \mathrm{~km}$, longitude of $\sim 140.7^{\circ}$ east, covering East Asia and Western Pacific regions [18]. The primary instrument aboard Himawari8, AHI, is a 16channel multispectral imager that captures visible and infrared images of the Asia-Pacific region [19]. AHI uses two scan mirrors and can produce images with a resolution down to $500 \mathrm{~m}$ and can provide a full disk observation every 10 minutes. AHI instrument features higher spatial, spectral, and radiometric resolution than its predecessors, in addition to shortened scanning times (about 10 minutes to image the Earth's full disk). It has 3 visible, 3 nearinfrared, and 10 infrared bands. The thermal infrared band's nadir spatial resolution is $2 \mathrm{~km}$. The TEB are calibrated using on-board blackbody and cold reference using deep space view. The instrument shows a significant nonlinear response and hence a quadratic response function is used for the calibration. The linear coefficient of the response function for each channel is calibrated every 10 minutes using the nonlinear coefficients derived from pre-launch testing [20].

\subsection{Spectral matching bands}

The MODIS and AHI matching bands are listed in Table 1 and their spectral response functions are shown in Figure 1. A double difference method in this work uses AHI as a bridge to facilitate a comparison between Terra and Aqua MODIS. Hence, the effect of differences in spectral response function between MODIS and AHI are insignificant to the first order. However, for a specific site, the spectral mismatch can have an effect that can change seasonally or with observation time. The analysis in this work uses the averaged results over the entire year to minimize this effect. For most of the matching bands, AHI bands have a broader spectral response function than MODIS. Four matching MODIS bands, 20 to 23, are compared with AHI band 7 centered at $3.9 \mu \mathrm{m}$. 
Table. 1 The center wavelengths for the matching bands of MODIS and AHI.

\begin{tabular}{|c|c|c|c|c|c|c|c|c|c|c|c|}
\hline MODIS Band & 20 & 21 & 22 & 23 & 27 & 28 & 29 & 30 & 31 & 32 & 33 \\
\hline MODIS Wavelength $(\mu \mathrm{m})$ & 3.78 & 3.97 & 3.97 & 4.05 & 6.77 & 7.34 & 8.53 & 9.73 & 11.02 & 12.03 & 13.36 \\
\hline AHI Band & \multicolumn{3}{|c|}{7} & 9 & 10 & 11 & 12 & 14 & 15 & 16 \\
\hline AHI Wavelength $(\mu \mathrm{m})$ & \multicolumn{3}{|c|}{3.9} & 6.9 & 7.3 & 8.6 & 9.6 & 11.2 & 12.4 & 13.3 \\
\hline
\end{tabular}
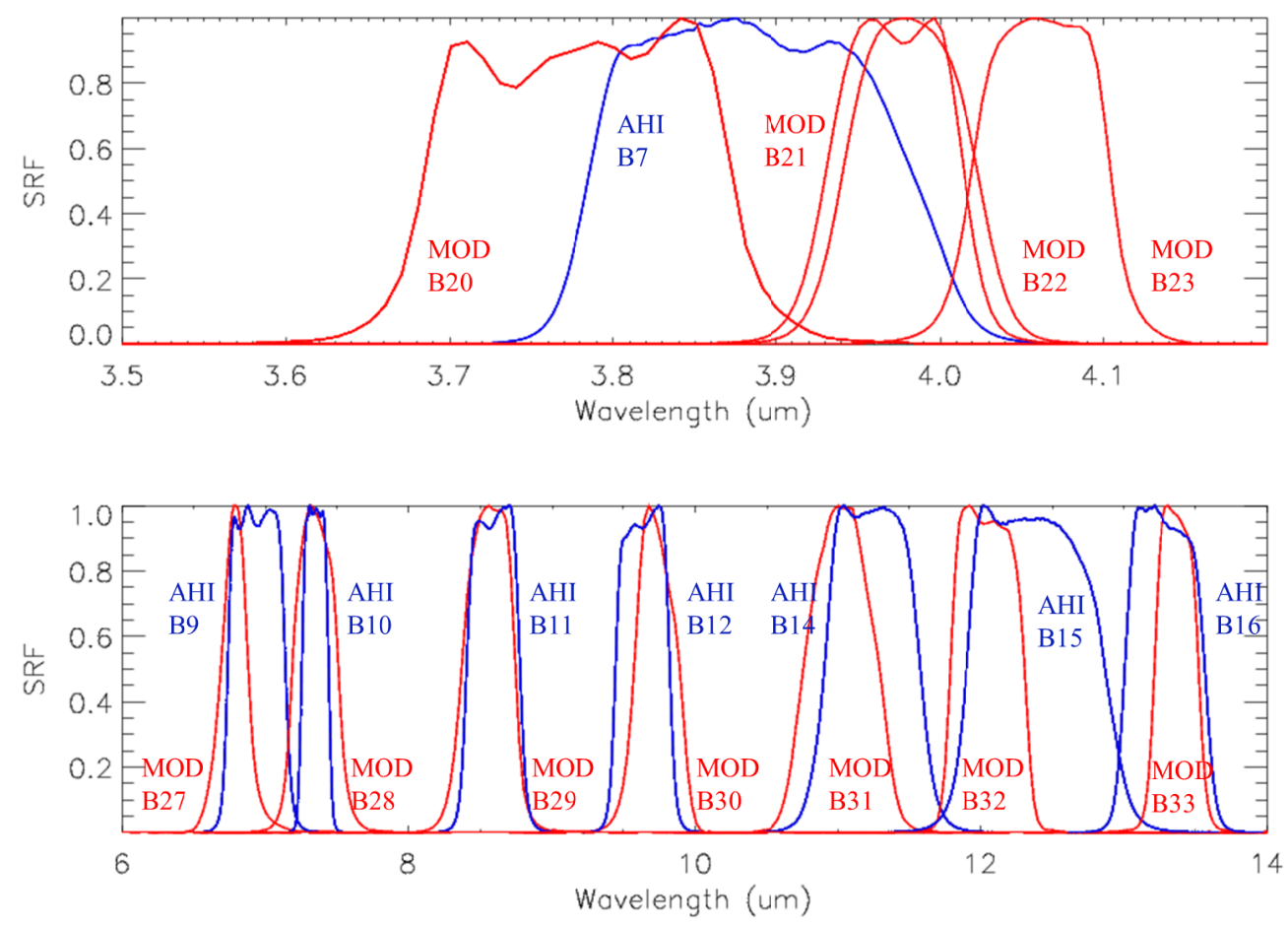

Figure 1. The spectral response function of the selected bands of MODIS (red curves) and AHI (blue curves).

\section{COMPARISON}

\subsection{Methodology development}

\subsubsection{Site selection and resampling}

The BT varies with time of the day and solar angle. The observation time difference also introduces impacts due to presence of cloud and varying atmospheric conditions. Hence, the comparisons should be performed with minimal time difference between the two overpasses. MODIS orbits the Earth and passes over a selected site once in daytime and once in nighttime every 1-2 days. AHI images the Earth's full disk every 10 minutes. It provides the feasibility to shorten the time difference between MODIS and AHI over selected site. For this study, the longest observation time difference is about 5 minutes. Since the double difference method is applied, this effect is reduced. Table 2 lists the selected sites used in this analysis. The site under AHI nadir is an ocean site and another desert site (Strzelecki Desert) in Australia are selected. The table also lists the latitudes and longitudes of the two sites. 
Table. 2 The latitude and Longitude ranges of the calibration sites

\begin{tabular}{|l|l|l|l|l|}
\hline Site & Lat_min & Lat_max & Lon_min & Lon_max \\
\hline AHI nadir(Ocean) & -1.00 & 1.00 & 139.70 & 140.70 \\
\hline Strzelecki Desert & -30.5 & -28.5 & 138.8 & 140.8 \\
\hline
\end{tabular}

\subsubsection{Pixel resampling}

The comparison in this work is pixel-to-pixel comparison between MODIS and AHI. However, the AHI pixel size is $2 \mathrm{~km}$ and MODIS pixel size at nadir is $1 \mathrm{~km}$. In addition, the geolocation error and pixel mismatch should be considered. The site should ideally be uniform and stable over different seasons. The MODIS measurements with different view angle range are used for comparison and correction of view angle dependence is applied. Table 2 lists the geolocation of the site.

The L1B data from MODIS and AHI are processed for comparison. The MODIS pixel size and orientation are different from those of AHI. There are two methods for the comparison on the selected site. One is to average the measurement of entire site and then perform the comparison. The second method is to resample the measurement and to perform the pixel-to-pixel comparison. The first method is simpler, but may introduce additional uncertainty. In this paper, a pixel-to-pixel comparison, after resampling, is used. The MODIS data are resampled and interpolated to the AHI grid over the selected area. The scene uniformity (also impacted by the presence of clouds) is considered in site selection. The pixel-to-pixel comparison after resampling enhances the comparison accuracy and also allows the comparison over a broad BT range.

\subsubsection{Uniformity and cloud screening}

The spatial resolution for AHI TEB is $2 \mathrm{~km}$, while it is $1 \mathrm{~km}$ (at nadir) for MODIS. The data resampling and sensor spatial uncertainty possibly reduces the comparison accuracy while viewing a non-uniform scene. The observation time difference also has effects due to presence of clouds and varying atmospheric conditions. MODIS level 2 products (MOD35 for Terra and MYD35 for Aqua) are used for identifying cloudy and clear sky measurements [21]. The cloud mask has four values indicating cloudy, partial cloudy, partial clear, clear. All pixels classified as totally clear are selected for this comparison. The scene uniformity is assessed using the cloud mask of surrounding pixels. A pixel selected for comparison should have same cloud mask classification over the $3 \times 3$ resampled AHI pixels. Additional uniformity filtering is also applied by using the variation of the nine surrounding $3 \times 3$ AHI pixels. Due to the modulation transfer function (MTF) effects, a uniform area around the pixel can enhance the comparison accuracy.

The clear sky ocean scene under AHI nadir direction provides a narrow BT range. Therefore, the BT dependency of Terra-Aqua differences cannot be comprehensively evaluated. Also, the selected ocean site has far fewer clear sky pixels. This double difference based comparison will also be applied to cloudy pixels to analyze Terra-Aqua difference at low BT in our future work. On the other hand, the desert site has more clear sky pixels which enhance the comparison accuracy. The desert site is located to the south of AHI nadir and has a fixed view angle. The desert site provides a larger range of ground BT and much more clear sky pixels for comparison. A uniformity filtering is applied to avoid the impacts due to pixel mismatch. In this work, the Terra-Aqua difference is the average of the difference over the BT range. In our future work, the BT dependency will also be analyzed.

\subsubsection{Correction and comparison}

The spectrally matching bands of AHI are used as a bridge for the comparison between the MODIS instruments. Figure 2 shows a flow chart for the MODIS comparison using AHI as bridge. The analysis of cloud effect reveals that the clear sky measurements are more stable and accurate for the comparison between MODIS and AHI. In addition, the MODIS measurement varies with view angle due to the absorption path difference. In the case of AHI, the ocean site is in nadir direction and the measurements do not have this effect. The view angle dependence can be derived using an empirical model. The Strzelecki Desert site is located at south of AHI nadir and has a non-zero view angle for one of the two scan mirrors. However, this view angle effect is fixed for the Terra-AHI and AquaAHI scene-pairs. For both sites, an empirical model is used for both Terra-AHI difference and Aqua-AHI difference. 


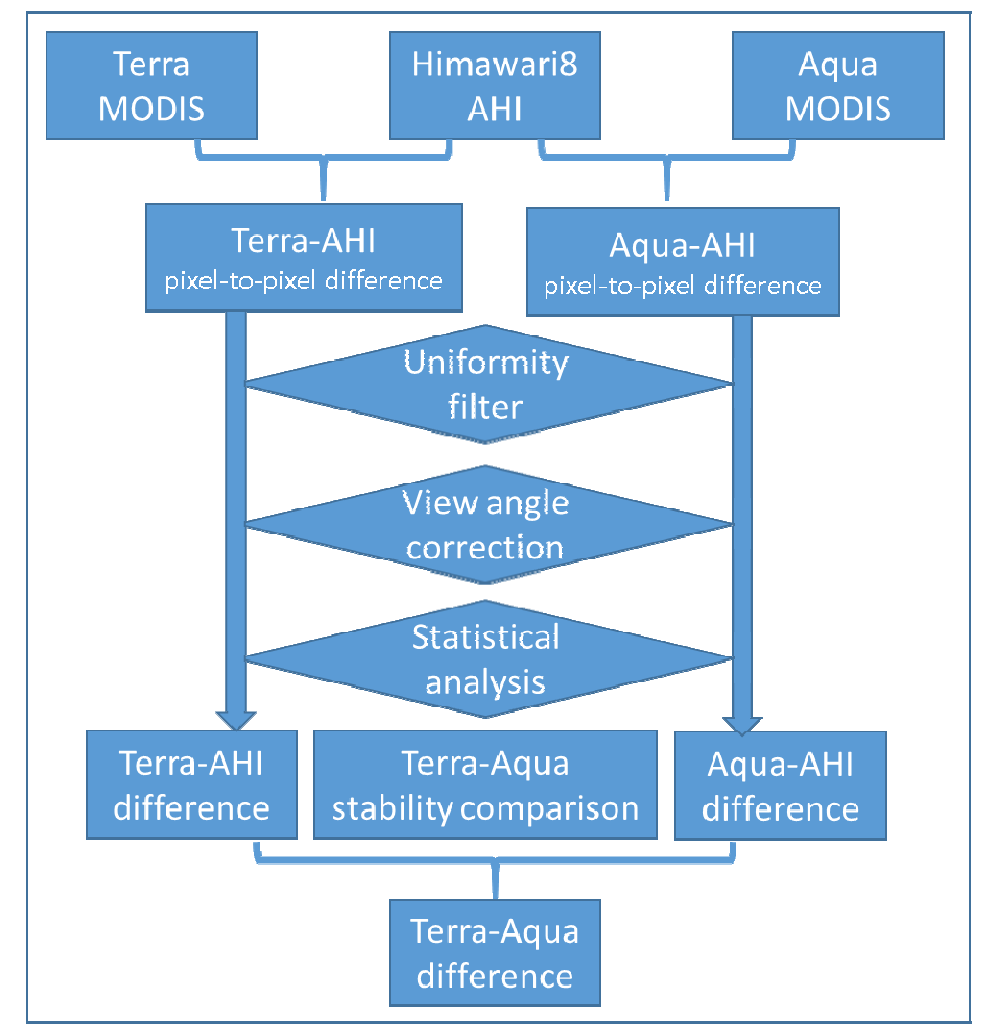

Figure 2. Flow chart for the MODIS comparison using AHI as a bridge.

\subsection{Comparison results}

\subsubsection{MODIS-AHI difference}

In this work, the averaged comparison difference from the year 2017 are analyzed. The one-year period can smooth the fluctuation and enhance the accuracy. The co-located L1B data with closest observation time for AHI and MODIS are collected and processed using the method shown in Figure 2. The differences between AHI and Aqua MODIS and between AHI and Terra MODIS are processed for all the matching bands. Figure 3 shows two examples (MODIS bands 29 and 31) of the comparison results for MODIS with AHI for clear sky over daytime ocean site, nighttime ocean site, and nighttime desert site. MODIS Band 31 has been stable for both Terra and Aqua. Terra band 29 has electronic cross-talk issues and a cross-talk correction has been implemented in the C6.1 L1B product used in this work [17]. Due to the spectral difference between the matching bands of MODIS and AHI, a large BT difference is expected. The ocean measurements have a narrow BT range. However, the MODIS-AHI difference shows a significant spread. The desert site provides more clear sky pixels and also shows a smaller spread. The Desert covers large BT range during the entire year measurement. The MODIS-AHI difference also covers different range for these two sites. 

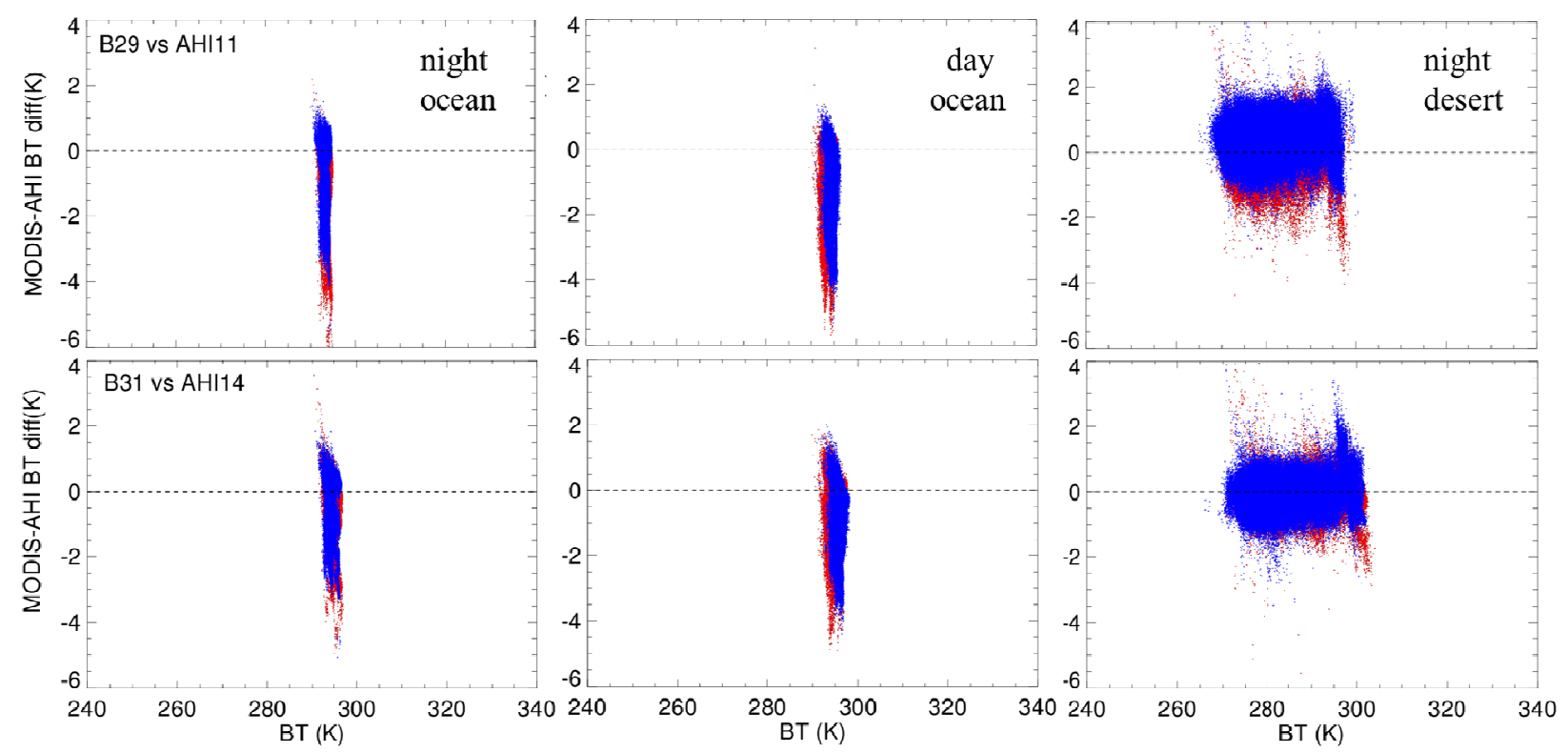

Figure 3. The MODIS-AHI difference using the ocean site under AHI nadir direction and Strzelecki Desert site. Top plots are for MODIS band 29 vs AHI band 11 and bottom plots are for band 31 vs AHI band 14. From left to right are for daytime ocean, nighttime ocean, and nighttime desert. The red symbols are the Terra-AHI difference and blue symbols are Aqua-AHI difference. The data used are the MODIS and AHI L1B data in year 2017.

\subsubsection{View angle dependency correction}

Even for the non-nadir desert site, the view angle dependency of AHI measurement is not an issue since it has the same bias for both Terra-AHI and Aqua-AHI difference. The bias can be removed in the double difference for Terra-Aqua comparison. The MODIS-AHI difference exhibits a view angle dependency. Before applying empirical model to perform the view angle correction, the symmetry of the measurement across the MODIS scan is evaluated. A symmetrical pattern of these differences around nadir is an indicator that the response versus scan angle (RVS) characterization exhibits reasonable accuracy. Figure 4 shows examples of MODIS-AHI difference as function of frame number (or MODIS scan angle). The red symbols are the Terra-AHI difference and blue symbols are AquaAHI difference. The difference are grouped as nighttime ocean, nighttime desert, and daytime desert.

After a few tests, an empirical model presenting the symmetry is applied. The model is expressed as

$$
\Delta T=c_{0}+c_{1}\left(f-f_{\text {nadir }}\right)^{2}+c_{2}\left(f-f_{\text {nadir }}\right)^{4}
$$

where $\Delta T$ is the MODIS-AHI BT difference, $f$ is the frame number in along scan direction, $f_{\text {nadir }}$ is the frame number corresponding to nadir. The coefficients $c_{0,1,2}$ are fitting parameters. $\left(f-f_{\text {nadir }}\right)$ is proportional to view angle. For the example showed in Figure 4, the pink line is the fitting for Terra-AHI difference and green is the fitting for Aqua-AHI difference. The corrected MODIS-AHI difference as function MODIS frame number is shown in Figure 5 for the scenes for bands 29 and 30. After correction, the nighttime measurements of these two sites are more stable than day time measurements. These view angle corrected MODIS-AHI differences are used to estimate the difference between the two MODIS instruments. 

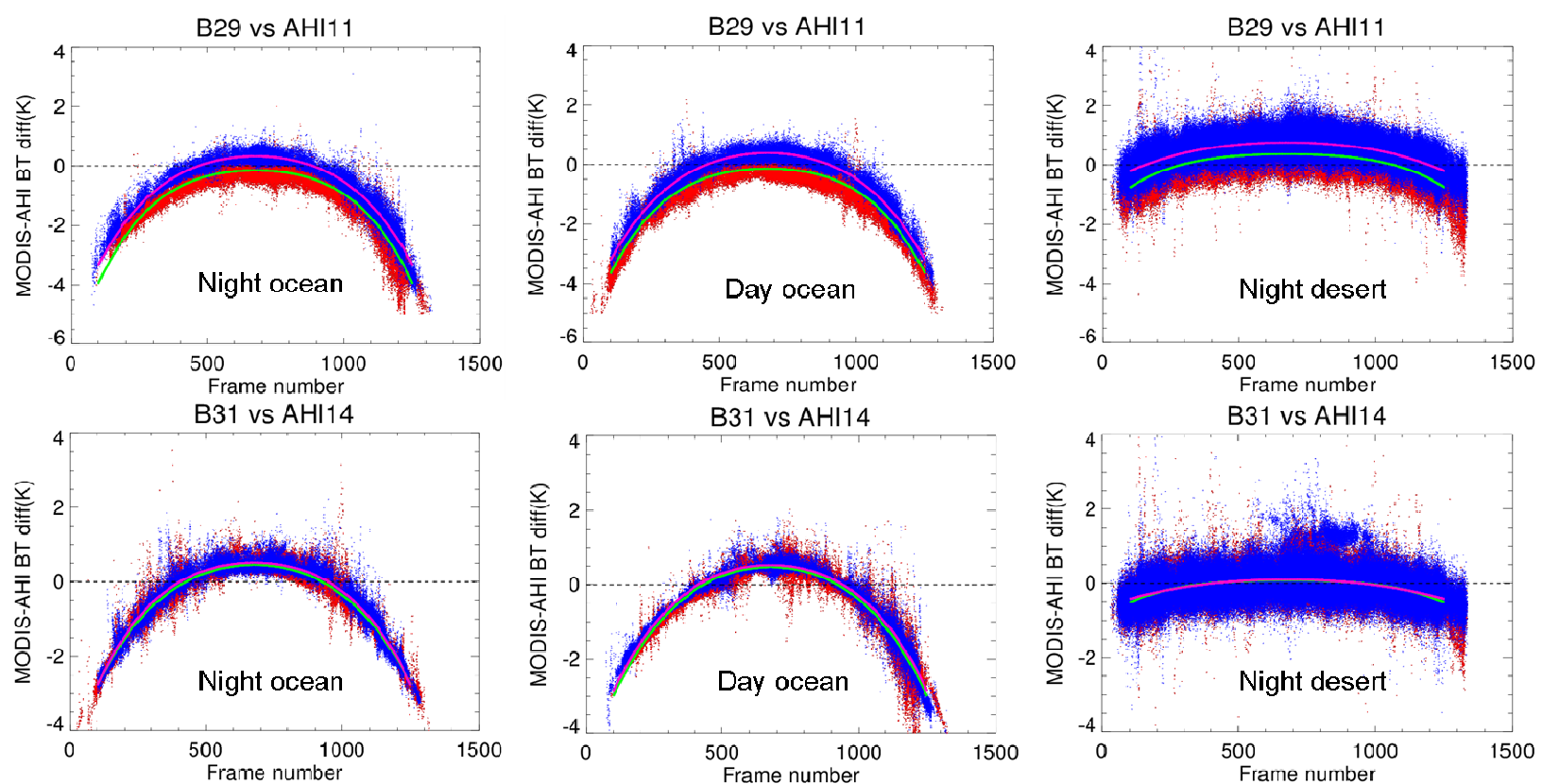

Figure 4. The MODIS-AHI difference as function MODIS frame number for bands 29 and 30 over three different scenes. The red symbols are the Terra-AHI difference and blue symbols are Aqua-AHI difference. The pink line is the fit using empirical model for Terra-AHI difference and green line is the fit for Aqua-AHI difference.
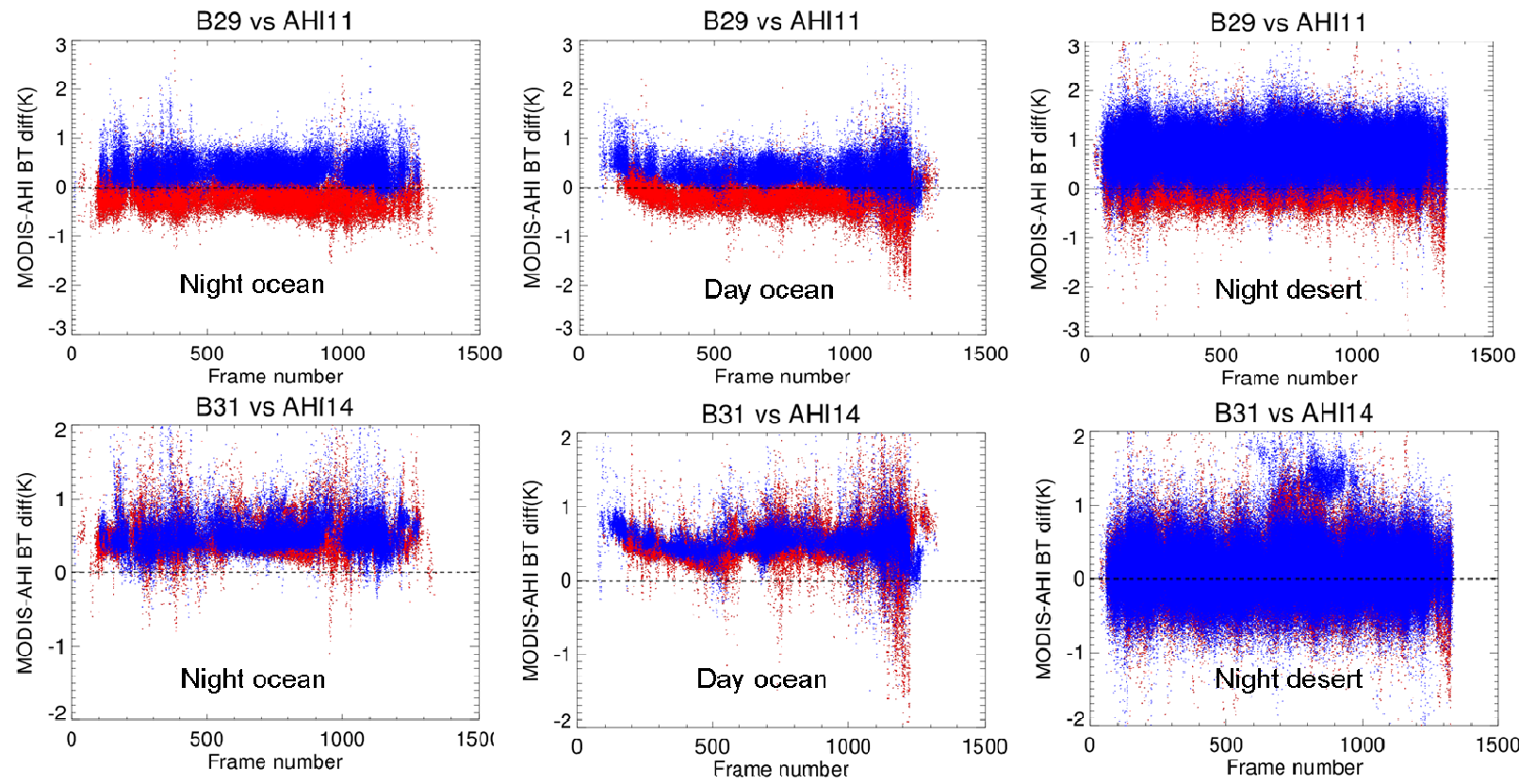

Figure 5. The corrected MODIS-AHI difference as function MODIS frame number for bands 29 and 30 over three different scenes. The red symbols are the Terra-AHI difference and blue symbols are Aqua-AHI difference.

\subsubsection{Terra and Aqua difference}

The comparison between AHI with Terra MODIS and AHI with Aqua MODIS shows larger difference for MODIS band 29 as compared to band 31. MODIS band 31 shows a more consistent comparison considering the uncertainty range. After view angle correction, a double difference is used to derive the Terra-Aqua difference. Table 3 lists the Terra-Aqua difference for different scenes. Only the results from nighttime measurement with clear sky are 
presented. The daytime effect and cloud issue are discussed in section 3.4.1. Except bands 21, 27, and 30, the difference is below $0.5 \mathrm{~K}$. Band 21 is a low gain band and the calibration use linear function [16]. Terra bands 27 to 30 have a known electronic cross-talk issue some residual effects post correction likely contribute to these disagreements [17]. Results from the Terra LWIR bands 27 to 30 are discussed in more detail in section 3.4.2.

Table 3 also lists the uncertainty of the comparison results. The uncertainty is only from the random effect and for the average difference from pixel-to-pixel comparison between instruments. If the uncertainty is completely due to random effect and the systematic errors are not included. The uncertainty of mean of the measurements is the uncertainty of single measurement divided by square root if number of samples. In this work, the entire year data are used and the number of samples are around 12000 for clear sky ocean and 55000 for clear sky desert. The uncertainty from the random effect is insignificant. The systematic errors are discussed in section 3.4, including the daytime effect and cross-talk effects.

Table 3. Terra-Aqua BT difference (K) for different scenes using two methods. For bands 20 to 23 over ocean site during day time, the Gaussian fit of MODIS-AHI difference failed and the results from that method are not valid.

\begin{tabular}{|l|l|c|c|c|c|c|c|c|c|c|c|c|}
\hline \multirow{2}{*}{ Scene } & AHI & 7 & 7 & 7 & 7 & 9 & 10 & 11 & 12 & 14 & 15 & 16 \\
\cline { 2 - 12 } & MODIS & 20 & 21 & 22 & 23 & 27 & 28 & 29 & 30 & 31 & 32 & 33 \\
\hline $\begin{array}{l}\text { Night } \\
\text { Ocean }\end{array}$ & Average (K) & 0.084 & -0.320 & 0.349 & 0.489 & 0.554 & -0.437 & -0.378 & -1.755 & -0.095 & 0.009 & 0.282 \\
\cline { 2 - 13 } & Uncertainty (K) & 0.001 & 0.006 & 0.001 & 0.001 & 0.005 & 0.001 & 0.001 & 0.002 & 0.001 & 0.001 & 0.002 \\
\hline \multirow{2}{*}{$\begin{array}{l}\text { Night } \\
\text { Desert }\end{array}$} & Average (K) & 0.142 & -0.881 & 0.293 & 0.308 & -0.019 & -0.286 & -0.222 & -0.688 & -0.047 & 0.021 & 0.119 \\
\cline { 2 - 13 } & Uncertainty (K) & 0.001 & 0.004 & 0.001 & 0.001 & 0.002 & 0.001 & 0.001 & 0.001 & 0.001 & 0.001 & 0.001 \\
\hline
\end{tabular}

\subsection{Discussion}

\subsubsection{Day time and cloud effects}

For the assessment of TEB performance, the nighttime measurements have an advantage, such as low impacts due to reflectance of sun light and cross-talk for the MWIR bands. As an example, Figure 6 shows the day time clear sky results for band 22. The empirical model cannot be applied to the MODIS-AHI difference as function of frame number. The two MODIS instruments have different overpass time and the ground BT range is also different. Hence, the large BT range difference can affect the Terra-AHI and Aqua-AHI in different ways and this bias can propagate to impact the Terra-Aqua comparison. The other bands, especially for MWIR bands, have similar effect and other issues on comparison using daytime measurements. In table 3, the Terra-Aqua comparison results are presented using nighttime measurements.
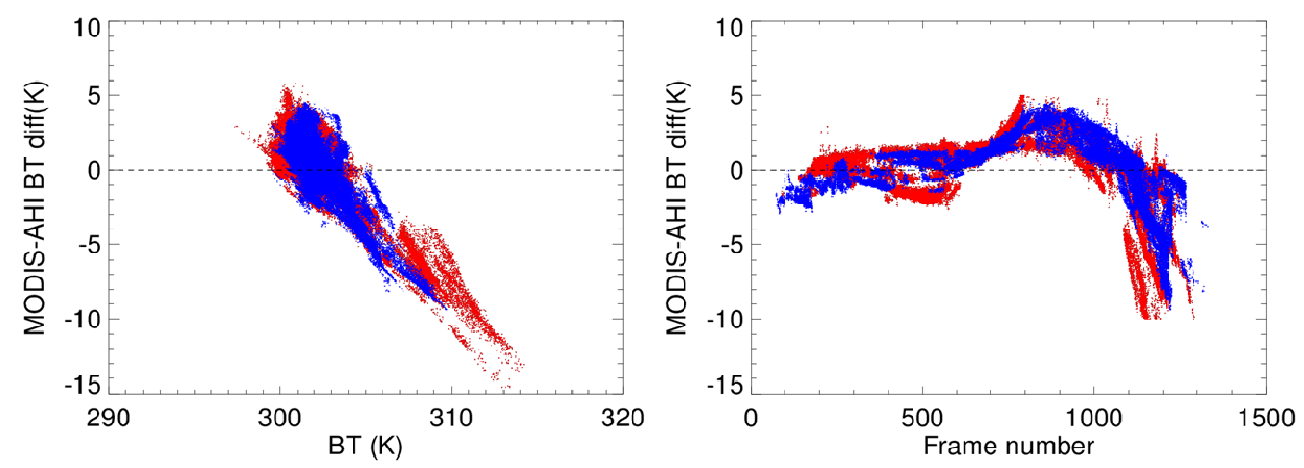

Figure 6. (Left) The MODIS-AHI difference as function of scene BT for band 22 over day time clear sky ocean. (Right) The MODIS-AHI difference as function of MODIS frame number for band 22 over day time clear sky ocean. The red symbols are the Terra-AHI difference and blue symbols are Aqua-AHI difference. 


\subsubsection{Terra PV LWIR cross-talk impact}

Terra has an electronic cross-talk issue for LWIR bands 27 to 30 [13]. The cross-talk correction has been applied to Collection 6.1 L1B which is used in this work. The cross-talk coefficients are derived from scheduled lunar observation and are updated if the coefficients change is beyond a certain limit. The residual of cross-talk effect and coefficient variations can also impact Terra L1B data. As shown in table 3, Terra bands 27 to 30 have large bias comparison with Aqua, up to $1.76 \mathrm{~K}$ for band 30 . It also shows that the biases are scene dependent. They are smaller for desert scene and larger for ocean scene. It is known that that cross-talk has large impact on Terra band 30 L1B product. The contamination is scene dependent and is also related to atmospheric conditions. For band 30, the contamination is the cross-talk from sending bands 27 to 29 and the bias is $1.76 \mathrm{~K}$ over ocean and $0.69 \mathrm{~K}$ over desert.

\section{SUMMARY}

A double difference method is applied to assess the differences in BT measurements between the two MODIS instruments using the geostationary imager, Himawari8/AHI. Ten thermal emissive bands of MODIS have spectrally overlapping bands with AHI. The site selected is an ocean area around the Himawari8 sub-orbital point and Strzelecki Desert located at south of Himawari8 sub-orbital point. The methods of data resampling have been applied to enhance the comparison accuracy. The time difference between the measurements from AHI and MODIS is less than 5 minutes. The use of clear sky scenes reduces the impact due to observation time difference. The comparison is performed using year 2017 collection 6.1 L1B data for MODIS. The view angle dependency has been observed in the MODIS-AHI difference. An empirical model is applied to remove the view angle dependency for both Terra and Aqua MODIS. The corrected MODIS-AHI differences are used for the comparison between Terra and Aqua using a double difference. Except bands 21, 27, and 30, the differences are less than 0.5K. Bands 27 and 30 have the contamination from cross-talk and the differences are scene dependent. Band 27 has $0.55 \mathrm{~K}$ difference for clear sky ocean and insignificant difference for clear sky desert. Band 30 has $1.76 \mathrm{~K}$ difference for clear sky ocean and $0.69 \mathrm{~K}$ difference for desert between the two MODIS instruments. This GEO-LEO double difference method can also apply to comparison of LEO-LEO instruments, such as between Visible Infrared Imaging Radiometer Suite (VIIRS) on SNPP and VIIRS on NOAA20, and also apply to comparison of GEO-GEO instruments, such as between Advanced Baseline Imager (ABI) on GOES16, AHI on Himawari8, and other similar GOES sensors.

\section{ACKNOWLEDGMENT}

This work is supported by the NASA MODIS Characterization Support Team (MCST) and NOAA/NASA GOES-R project. The Himawari-8/AHI L1B data was supplied by the P-Tree System, Japan Aerospace Exploration Agency (JAXA).

\section{REFERENCES}

[1] Xiong, X., A. Wu, B. N. Wenny, S. Madhavan, Z. Wang, Y. Li, N. Chen, W. Barnes, and V. Salomonson, "Terra and Aqua MODIS Thermal Emissive Bands On-Orbit Calibration and Performance", IEEE Transactions on Geoscience and Remote Sensing, vol. 53, issue 10, pp. 5709 - 5721, 2015.

[2] Xiong, X., A. Angal, A. Wu, Z. Wang, W. Barnes, and V. Salomonson, "15 Years of Aqua MODIS OnOrbit Operation, Calibration, and Performance", IEEE 2017 International Geoscience \& Remote Sensing Symposium, pp. 4695-4698, 2017

[3] Cavalli, R.M, "Retrieval of Sea Surface Temperature from MODIS Data in Coastal Waters.", Sustainability, 9(11), 2032, 2017

[4] Sobrino, J.A., J. El Kharraz, and Z. L. Li, "Surface temperature and water vapour retrieval from MODIS data. Int. J. Remote Sens., 24, 5161-5182, 2003

[5] Moeller, C., R. Frey, E. Borbas, W. P. Menzel, T. Wilson, A. Wu, and X. Geng, "Improvements to Terra MODIS L1B, L2, and L3 science products through using crosstalk corrected L1B radiances", Proc. SPIE 10402, Earth Observing Systems XXII, $1040200 \quad$ (5 September 2017); doi: 10.1117/12.2274340; https://doi.org/10.1117/12.2274340 
[6] Yue, H., C. He, Y. Zhao, Q. Ma, and Q. Zhang, "The brightness temperature adjusted dust index: An improved approach to detect dust storms using MODIS imagery". INTERNATIONAL JOURNAL OF APPLIED EARTH OBSERVATION AND GEOINFORMATION, 57, 166-176, 2017.

[7] Skakun, S., C. O. Justice, E. Vermote, and J. C. Roger, Transitioning from MODIS to VIIRS: an analysis of inter-consistency of NDVI data sets for agricultural monitoring" International journal of remote sensing, 39(4), 971992, 2018

[8] Gupta, P., L. A. Remer, R. C. Levy, and S. Mattoo, "Validation of MODIS $3 \mathrm{~km}$ land aerosol optical depth from NASA's EOS Terra and Aqua missions" Atmospheric Measurement Techniques; Katlenburg-Lindau 11 (5), 2018

[9] Levy, R. C., S. Mattoo, L. A. Munchak, L. A. Remer, A. M. Sayer, F. Patadia, and N. C. Hsu, "The Collection 6 MODIS aerosol products over land and ocean", Atmos. Meas. Tech., 6, 2989-3034, 2013

[10] Hulley, C. G., N. K. Malakar, T. Islam, and R. J. Freepartner, "NASA's MODIS and VIIRS Land Surface Temperature and Emissivity Products: A Long-Term and Consistent Earth System Data Record", EEE Journal of Selected Topics in Applied Earth Observations and Remote Sensing, 11(2), 522-535, 2018

[11] Toure, A.M., R. H. Reichle, B. A. Forman, A. Getirana, and, G.J.M. Lannoy, "Assimilation of MODIS Snow Cover Fraction Observations into the NASA Catchment Land Surface Model", Remote Sens. 10(316), 2018

[12] Xiong, X., C. Cao, and G. Chander, "An Overview of Sensor Calibration Inter-comparison and Applications", Frontiers of Earth Science in China, vol. 4, issue 2, pp. 237-252, 2010

[13] Chander, G., T. J. Hewison, N. Fox, X. Wu, X. Xiong, and W. J. Blackwell, "Overview of Intercalibration of Satellite Instruments", IEEE TRANSACTIONS ON GEOSCIENCE AND REMOTE SENSING, 51(3), 10562013

[14] Cao, C., M. Weinreb, and H. Xu, "Predicting simultaneous nadir overpasses among polar-orbiting meteorological satellites for intersatellite calibration of radiometers", Journal of Atmospheric and Oceanic Technology, vol. 21, pp. 537-542, 2004.

[15] Xiong, X., A. Wu, and C. Cao, "On-orbit calibration and inter-comparison of Terra and Aqua MODIS surface temperature spectral bands", International Journal of Remote Sensing, 29: 5347-5359, 2008

[16] Xiong, X., K. Chiang, B. Guenther, and W. Barnes, "MODIS Thermal Emissive Bands Calibration Algorithm and On-orbit Performance", Proceedings of SPIE - Optical Remote Sensing of the Atmosphere and Clouds III, 4891, 392-401, 2002.

[17] Wilson, T., A. Wu, A. Shrestha, X. Geng, Z. Wang, C. Moeller, R. Frey, and X. Xiong, "Development and implementation of an electronic crosstalk correction for bands 27-30 in Terra MODIS collection 6," Remote Sens. 9(6), 569 (2017).

[18] Bessho, K., M. Hayashi, A. Ikeda, H. Inoue, Y. Kumagai, T. Miyakawa, H. Murata, O. Tomoo, A. Okuyama, R. Oyama, et al., "An introduction to Himawari8/9japans new-generation geostationary meteorological satellites," Journal of the Meteorological Society of Japan. Ser. II 94(2), 151-183, 2016.

[19] Griffith, P., "Advanced Himawari Imager (AHI) design and operational flexibility", 6th Asia/Oceania Meteorological Satellite Users' Conference, 10 November 2015.

[20] Okuyama, A., A. Andou, K. Date, N. Mori, H. Murata, T. Tabata, M. Takahashi, R. Yoshino, and K. Bessho, "Preliminary validation of Himawari-8/AHI navigation and calibration", 6th Asia/Oceania Meteorological Satellite Users' Conference, Nov., 2015.

[21] Platnick, S., M. D. King, S. A. Ackerman, W. P. Menzel, B. A. Baum, J. C. Riédi, and R. A. Frey, "The MODIS Cloud Products: Algorithms and Examples From Terra", IEEE Transactions On Geoscience And Remote Sensing, 41(2), 459-473, 2003. 